\title{
MicroRNA-148a inhibits the proliferation and promotes the paclitaxel-induced apoptosis of ovarian cancer cells by targeting PDIA3
}

\author{
SHUZHEN ZHAO ${ }^{1}$, ZHENGFANG WEN ${ }^{1}$, SHANSHAN LIU ${ }^{1}$, YING LIU ${ }^{1}$, \\ XIAORUI LI ${ }^{2}$, YANNA GE ${ }^{1}$ and SHAORU LI ${ }^{1}$ \\ Departments of ${ }^{1}$ Gynecology and Obstetrics, and ${ }^{2}$ Oncology, \\ The First Affiliated Hospital of Xinxiang Medical University, Weihui, Henan 453100, P.R. China
}

Received July 31, 2014; Accepted April 16, 2015

DOI: $10.3892 / \mathrm{mmr} .2015 .3826$

\begin{abstract}
MicroRNAs (miRs) are a class of non-coding RNAs that function as key regulators of gene expression at the post-transcriptional level. miR-148a has been suggested to be associated with human ovarian cancer, however, the detailed functions of miR-148a in ovarian cancer remain to be fully elucidated. The present study aimed to investigate the regulatory mechanism of miR-148a in ovarian cancer cells. Reverse transcription-quantitative polymerase chain reaction and western blot analysis were conducted to examine the RNA and protein levels, respectively. The luciferase reporter assay was used to determine the target relationship. Cell proliferation and apoptosis assays were additionally conducted. The present study demonstrated that miR-148a inhibited cell proliferation and promoted the paclitaxel-induced apoptosis of ovarian cancer cells. Furthermore, protein disulfide isomerase family $\mathrm{A}$, member 3 (PDIA3) was identified as a target gene of miR-148a. A fluorescent reporter assay was performed to confirm that miR-148a was able to directly bind to the 3'-untranslated region of PDIA3 mRNA. In addition, miR-148a was frequently downregulated in ovarian cancer tissue, whereas the expression levels of PDIA3 were increased. Knockdown of PDIA3 significantly inhibited the proliferation and promoted the paclitaxel-induced apoptosis of the ovarian cancer cells, whereas overexpression of PDIA3 had the opposite effects. Therefore, the results of the present study suggested that miR-148a inhibited the proliferation and promoted the paclitaxel-induced apoptosis of ovarian cancer cells, and this may be partly attributed to direct targeting of PDIA3.
\end{abstract}

Correspondence to: Dr Shaoru Li, Department of Gynecology and Obstetrics, The First Affiliated Hospital of Xinxiang Medical University, 88 Jiankang Road, Weihui, Henan 453100, P.R. China E-mail: xinxianglishaoru@163.com

Key words: ovarian cancer, microRNA-148a, paclitaxel, apoptosis, protein disulfide isomerase family A, member 3

\section{Introduction}

Ovarian cancer is one of the most common types of solid tumor in females, and is associated with a poor prognosis and high mortality rate $(1,2)$. Accumulating evidence has demonstrated that oncogenes and tumor suppressors may be important in the development and progression of ovarian cancer $(3,4)$. However, the underlying molecular mechanisms remain to be fully elucidated.

MicroRNAs (miRs) are a class of small, non-coding RNAs, which can negatively regulate gene expression, either through translational suppression of their target mRNAs, or the induction of target mRNA degradation (5). Several studies have demonstrated that the aberrant expression of miRNAs is associated with tumorigenesis in a temporal and spatial manner $(6,7)$. In addition, previous reports have indicated that miR-148a may act as a tumor suppressor in human malignancy $(8,9)$. miR-148a has been reported to the suppress epithelial-to-mesenchymal (ETM) transition by targeting ROCK1 in non-small cell lung cancer cells (9). Furthermore, miR-148a suppresses tumor cell invasion and metastasis by inhibiting ROCK1 in gastric cancer (10), and inhibits the ETM and metastasis of hepatoma cells by targeting Met/Snail signaling (11). Zhou et al (12) demonstrated that the expression of miR-148a is frequently downregulated in ovarian cancer tissue and cell lines, and upregulation of miR-148a inhibits the proliferation of ovarian cancer cells. These findings suggest that miR-148a may have a suppressive role in the regulation of ovarian cancer growth, however, the role of miR-148a, and its underlying mechanism have not been investigated previously.

The present study aimed to elucidate the role of miR-148a in the regulation of cell proliferation and apoptosis in ovarian cancer in vitro. In addition, the current study investigated the underlying mechanisms.

\section{Materials and methods}

Reagents. Dulbecco's modified Eagle's medium (DMEM), TRIzol ${ }^{\circledR}$ reagent, fetal bovine serum (FBS), Opti-MEM medium, miRNA Reverse Transcription kit, SYBR Ex 
Taq kit and Lipofectamine ${ }^{\circledR} 2000$ were purchased from Invitrogen Life Technologies (Carlsbad, CA, USA). An miRNA quantitative-polymerase chain reaction (qPCR) Detection kit was purchased from GeneCopoeia (Rockville, MD, USA). Monoclonal mouse anti-PDIA3 (1:100; ab13506), monoclonal mouse anti-GAPDH (1:50; ab8245) and rabbit anti-mouse secondary $\operatorname{IgG}(1: 5,000 ;$ ab175743) antibodies were purchased from Abcam (Cambridge, UK). The enhanced Chemiluminescence (ECL) kit was purchased from Pierce Biotechnology, Inc. (Rockford, IL, USA). The Quick-Change Site-Directed Mutagenesis kit was purchased from Agilent Technologies, Inc.(Santa Clara, CA, USA). The PsiCHECK ${ }^{\mathrm{TM}} 2$ vector was purchased from Promega Corporation (Madison, WI, USA). The Apoptosis Detection kit I was purchased from BD Biosciences (San Diego, CA, USA).

Tissue specimen collection. The present study was approved by the Ethics Committee of Xinxiang Medical University (Weihui, China). Cancerous tissue and matched normal adjacent tissue samples were obtained from 20 female ovarian adenocarcinoma patients (age, 35-65) of different TNM stages (T1, 4; T2, 5; T3, 6; T4, 5). These samples were collected at the Department of Gynecology and Obstetrics, the First Affiliated Hospital of Xinxiang Medical University. Informed consent was obtained from each of the patients with ovarian cancer. Following surgical removal, the tissue samples were immediately snap-frozen in liquid nitrogen until subsequent use.

Cell culture. The SKOV3 human ovarian cancer cell line was purchased from the Cell Bank of Type Culture Collection of Chinese Academy of Sciences, Shanghai Institute of Cell Biology (Shanghai, China). The cells were cultured in DMEM supplemented with $10 \% \mathrm{FBS}, 100 \mathrm{IU} / \mathrm{ml}$ penicillin and $100 \mathrm{mg} / \mathrm{ml}$ streptomycin (Sigma-Aldrich). The cells were incubated at $37^{\circ} \mathrm{C}$ in a humidified chamber containing $5 \% \mathrm{CO}_{2}$. Paclitaxel (100 nM; Sigma-Aldrich) was used to treat SKOV3 cells to induce cell apoptosis.

Reverse transcription (RT)-qPCR. Total RNA was extracted from the tissues and the cells using TRIzol ${ }^{\circledR}$ reagent, according to the manufacturer's instructions. Tissues were frozen in liquid nitrogen and were homogenized. The integrity of the large RNAs was confirmed using $1 \%$ denatured agarose gel (Sigma-Aldrich) electrophoresis. RT-qPCR was performed in order to detect the expression levels of miR-148a. In accordance with the manufacture's instructions, the RNA was reverse transcribed into cDNA using a miRNA Reverse Transcription kit. The cDNA (10 ng) was then used for amplification of mature miR-148a, and U6 snRNA was used as an endogenous control. qPCR was performed using an miRNA q-PCR Detection kit on an ABI 7500 thermocycler (ABI 7500; Applied Biosystems Life Technologies, Foster City, CA, USA), according to the manufacturer's instructions. The primers from Shanghai Shenggong Co., Ltd. (Shanghai, China) used were as follows: PDIA3, forward 5'-GCCTCCGACGTGCTAGAAC-3' and reverse 5'-GCGAAGAACTCGACGAGCAT-3'; GAPDH, forward 5'-GGAGCGAGATCCCTCCAAAAT-3' and reverse 5'-GGCTGTTGTCATACTTCTCATGG-3'.
The PCR cycling conditions were as follows: $94^{\circ} \mathrm{C}$ for $3 \mathrm{~min}$, followed by 40 cycles at $94^{\circ} \mathrm{C}$ for $30 \mathrm{sec}, 56^{\circ} \mathrm{C}$ for $30 \mathrm{sec}$ and $72^{\circ} \mathrm{C}$ for $30 \mathrm{sec}$. To determine the mRNA expression levels of PDIA3, an SYBR Ex Taq kit was used for the PCR amplification of PDIA3. GAPDH was used as the endogenous control gene. The PCR cycling conditions were as follows: $94^{\circ} \mathrm{C}$ for $3 \mathrm{~min}$, followed by 40 cycles at $94^{\circ} \mathrm{C}$ for $30 \mathrm{sec}, 60^{\circ} \mathrm{C}$ for $30 \mathrm{sec}$ and $72^{\circ} \mathrm{C}$ for $30 \mathrm{sec}$. The relative expression levels were analyzed using the $2^{-\Delta \Delta \mathrm{Ct}}$ method (13).

Transfection. Transfection was performed using Lipofectamine ${ }^{\circledR} 2000$, according to the manufacturer's instructions. At the time of transfection, the cells were $\geq 70 \%$ confluent. The oligonucleotides (scrambled miRNA, miR-148a mimic, miR-148a inhibitor, PDIA3 specific siRNA) and plasmids (PDIA3) were incubated in Opti-MEM medium at $37^{\circ} \mathrm{C}$ for $48 \mathrm{~h}$. For miR-148a functional analysis, the SKOV3 cells $\left(1 \times 10^{6}\right)$ were transfected with the scrambled miRNA as a negative control (NC), the miR-148a mimic or the miR-148a inhibitor (Invitrogen Life Technologies). For PDIA3 functional analysis, the SKOV3 cells $\left(1 \times 10^{6}\right)$ were transfected with PDIA3-specific smallinterfering (si)RNA or a PDIA3 plasmid (GenePharma Co., Ltd., Shanghai, China).

Bioinformatics. The miRNA targets were identified using the TargetScan database (http://www. targetscan.org).

Fluorescent reporter assay. A Quick-Change Site-Directed Mutagenesis kit was used to generate a mutant 3'-untranslated region (UTR) of PDIA3, according to the manufacturer's instructions. The wild type or mutant 3'-UTRs of PDIA3 were inserted into the psiCHECK ${ }^{\mathrm{TM}} 2$ vector by performing restriction enzyme digestion using XhoI and EcoRI (New England BioLabs, Ipswich, MA, USA), then ligation using T4 DNA ligase (New England BioLabs). The vectors were transfected into SKOV3 cells using Lipofectamine ${ }^{\circledR} 2000$, according to the manufacturer's instructions. Cells were incubated at $37^{\circ} \mathrm{C}$ for $48 \mathrm{~h}$. Once the SKOV3 cells were cultured to $\sim 70 \%$ confluence, they were transfected with the psiCHECK ${ }^{\mathrm{TM}}$ 2-PDIA3-3'-UTR or psiCHECK ${ }^{\mathrm{TM}}$ 2-mutant PDIA3-3'-UTR vector, with or without $100 \mathrm{nM}$ miR-148a mimics, respectively. At $48 \mathrm{~h}$ post-transfection, the luciferase activities were determined using an LD400 luminometer (Beckman Coulter, Inc., Brea, CA, USA). The activity of Renilla luciferase was normalized to that of firefly luciferase activity.

MTT assay. The cells $\left(5 \times 10^{3}\right.$ cells/well) were seeded in a 96-well plate, $24 \mathrm{~h}$ after transfection. Following $48 \mathrm{~h}$ incubation at $37^{\circ} \mathrm{C}$, the cells were incubated with MTT $(0.5 \mathrm{mg} / \mathrm{ml}$; Sigma-Aldrich) at $37^{\circ} \mathrm{C}$ for $4 \mathrm{~h}$. The media was then removed, and the precipitated formazan (Sigma-Aldrich) was dissolved in $100 \mathrm{ml}$ dimethyl sulfoxide. The absorbance was measured at a wavelength of $570 \mathrm{~nm}$ (PHERAstar FS; Life Technologies, Carlsbad, CA, USA).

Apoptosis assay. The cells $\left(1 \times 10^{6}\right)$ were plated into 6-well plates. For each treatment group, flow cytometry (C6; BD Biosciences, Franklin Lakes, NJ, USA) was used to determine the apoptotic rate of the SKOV3 cells. Briefly, the relative quantities of annexin V-fluorescein isothiocyanate-positive/propidium 
iodide-negative cells were determined using an Apoptosis Detection kit I, according to the manufacturer's instructions.

Western blot analysis. Tissue samples and cells were lysed using radioimmunoprecipitation lysis buffer (Sigma-Aldrich), and the proteins were then harvested. The protein concentrations were subsequently determined using a Bicinchoninic Protein Assay Kit (Pierce Biotechnology, Inc.) according to the manufacturer's instructions. The polyvinylidene difluoride (PVDF; Life Technologies) membrane was blocked with phosphate-buffered saline (PBS; Sigma-Aldrich) supplemented with $5 \%$ non-fat milk. The protein $(\sim 25 \mu \mathrm{g})$ was separated using $12 \%$ SDS-denatured polyacrylamide gel electrophoresis, and transferred onto a PVDF membrane. The PVDF membrane was then incubated with mouse anti-PDIA3 and mouse anti-GAPDH antibodies, at room temperature for $3 \mathrm{~h}$. After being washed three times with PBS containing 5\% Tween (Sigma-Aldrich), the PVDF membrane was incubated with horseradish peroxidase-conjugated rabbit anti-mouse secondary antibody at room temperature for $1 \mathrm{~h}$. An ECL kit was used to visualize the blots. The relative protein expression levels were analyzed using Image-Pro Plus 6.0 software (Media Cybernetics, Inc., Rockville, MD, USA).

Statistical analysis. The results are expressed as the mean \pm standard deviation of three independent experiments. Statistical analysis of differences was assessed using a two-tailed Student's t-test. SPSS software, version 17.0 (SPSS, Inc., Chicago, IL, USA) was used for the statistical analysis. $\mathrm{P}<0.05$ was considered to indicate a statistically significant difference.

\section{Results}

miR-148a inhibits proliferation and promotes paclitaxel-induced apoptosis of ovarian cancer cells. The present study examined the effects of the upregulation or inhibition of miR-148a on the proliferation and paclitaxel-induced apoptosis of ovarian cancer cells. Following transfection of the SKOV3 cells with the miR-148a mimic or inhibitor, the expression levels of miR-148a were determined using RT-qPCR. As shown in Fig. 1A, transfection with the miR-148a mimic significantly upregulated the expression levels of miR-148a $(\mathrm{P}<0.01)$, whereas transfection with the miR-148a inhibitor significantly decreased the expression levels of miR-148a in the SKOV3 cells, compared with the control group $(\mathrm{P}<0.01)$. Following transfection for $72 \mathrm{~h}$, the viability of the cells in each group were determined using an MTT assay. As shown in Fig. 1B, the upregulation of miR-148a markedly inhibited the cell viability, whereas inhibition of miR-148a significantly promoted the cell viability, compared with the control group $(\mathrm{P}<0.01)$. These results indicated that miR-148a had an inhibitory effect on SKOV3 cell proliferation. In addition, paclitaxel (100 $\mathrm{nM})$ was used to treat the SKOV3 cells and induce cell apoptosis, and the percentages of apoptotic cells were detected $48 \mathrm{~h}$ following transfection. As shown in Fig. 1C, overexpression of miR-148a promoted paclitaxel-induced cell apoptosis $(\mathrm{P}<0.01)$, whereas inhibition of miR-148a suppressed paclitaxel-induced cell apoptosis, compared with the control group $(\mathrm{P}<0.01)$. These results indicated that miR-148a had a promoting effect on paclitaxel-induced apoptosis of ovarian cancer cells.

PDIA3 is a direct target gene of $m i R-148 a$. Downregulation of miR-148a may function as a tumor suppressor, and may suppress tumor growth by inhibiting target genes. Therefore, the present study performed bioinformatic analysis in order to predict the potential target genes of miR-148a, which regulate tumor cell growth. PDIA3 was identified as a possible target gene of miR-148a, which has been reported to be associated with several types of cancer, including ovarian cancer $(14,15)$. To investigate whether PDIA3 is a target of miR-148a, wild-type (WT) and mutant (MUT) PDIA3 3'-UTR were generated (Fig. 2A). A luciferase reporter assay was then performed in the SKOV3 ovarian cancer cells. As shown in Fig. 2B, the luciferase activity was significantly reduced in the SKOV3 cells co-transfected with the WT PDIA3 3'-UTR and miR-148a mimics $(\mathrm{P}<0.01)$. However, luciferase activity was unchanged in the SKOV3 cells, which were co-transfected with the MUT PDIA3 3'-UTR and miR-148a mimics, indicating PDIA3 as a target gene of miR-148a. Subsequently, the effects of miR-148a on the mRNA and protein expression levels of PDIA3 were evaluated in the SKOV3 cells. As shown in Figs. 2C and D, the mRNA and protein expression levels of PDIA3 were significantly reduced following overexpression of miR-148a $(\mathrm{P}<0.01)$, but increased following inhibition of miR-148a in the SKOV3 cells $(\mathrm{P}<0.01)$. These results indicated that miR-148a negatively regulated the expression of its target, PDIA3, in SKOV3 ovarian cancer cells.

miR-148a is inversely correlated with the expression of PDIA3 in ovarian cancer tissue. RT-qPCR was performed to determine the expression levels of miR-148a in 20 ovarian cancer tissue samples and matched adjacent normal tissue samples. As shown in Fig. 3A, the expression levels of miR-148a were significantly downregulated in ovarian cancer tissue, compared with the matched normal adjacent tissue. Subsequently, the mRNA expression levels of PDIA3 were examined in ovarian cancer and matched adjacent normal tissue. As shown in Fig. 3B, the mRNA expression levels of PDIA3 were markedly increased in the ovarian cancer tissue, compared with the matched normal adjacent tissue. Furthermore, a significant inverse correlation was observed between miR-148a and the expression of PDIA3 in the ovarian cancer tissue (Fig. 3C). These results suggested that upregulation of PDIA3 may be due to downregulation of miR-148a in ovarian cancer tissue.

PDIA3 promotes cell proliferation and inhibits paclitaxel-induced apoptosis in ovarian cancer cells. Based on the above findings, it was hypothesized that miR-148a inhibits proliferation and promotes paclitaxel-induced apoptosis of ovarian cancer cells through direct targeting of PDIA3. However, the effects of PDIA 3 on ovarian cancer cell proliferation and paclitaxel-induced apoptosis remain to be elucidated. To confirm this hypothesis, the SKOV3 cells were transfected with either a pcDNA3.1-PDIA3 plasmid or PDIA3 siRNA to upregulate or downregulate the expression of PDIA3, 

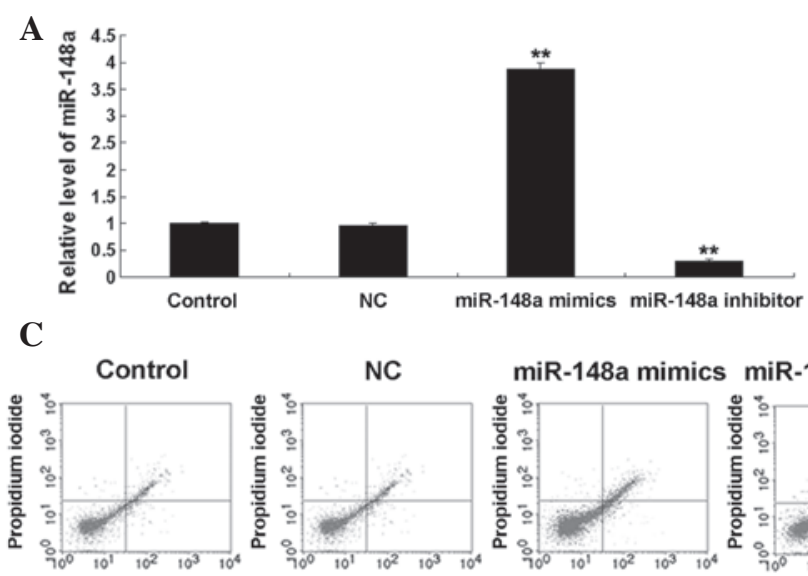

Annexin V-FITC

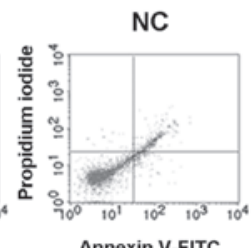

Annexin V-FITC

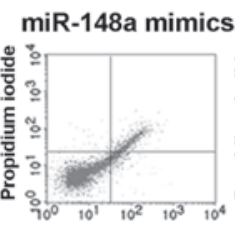

Annexin V-FITC

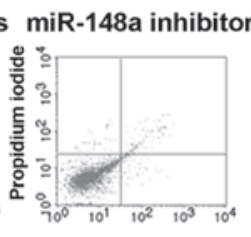

Annexin V-FITC
B
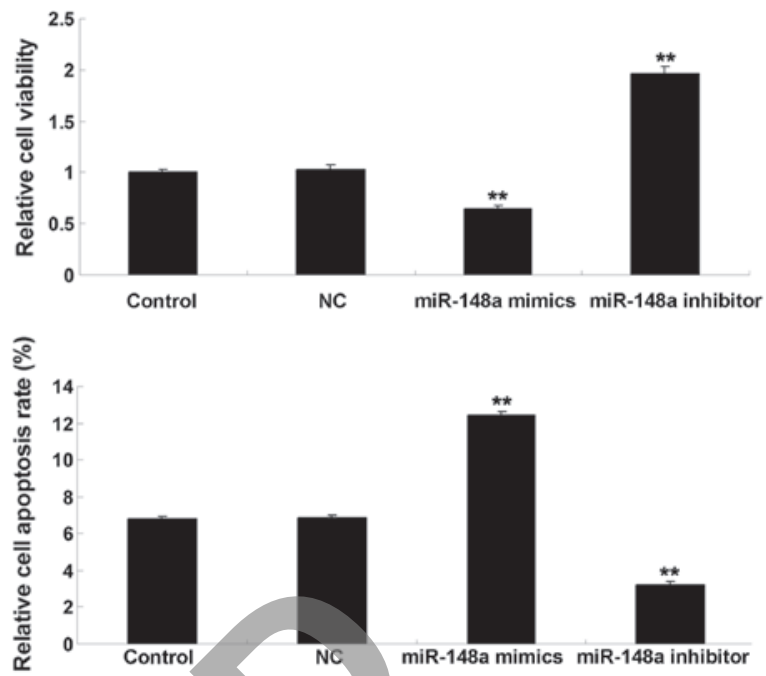

miR-148a mimics miR-148a inhibitor

Figure 1. (A) Transfection with the miR-148a mimic significantly upregulated the expression levels of miR-148a, whereas transfection with the miR-148a inhibitor significantly decreased the expression levels of miR-148a in the SKOV3 human ovarian cancer cells, compared with the control cells. ** $\mathrm{P}<0.01$, vs. control. (B) MTT assay demonstrated that miR-148a upregulation markedly inhibited cell viability, whereas miR-148a inhibition significantly promoted cell viability, compared with the control group. ${ }^{* *} \mathrm{P}<0.01$, vs. control. (C) Overexpression of miR-148a promoted paclitaxel-induced cell apoptosis, whereas inhibition of miR-148a suppressed paclitaxel-induced cell apoptosis, compared with the control group. ${ }^{*} \mathrm{P}<0.01$, vs. control. The results are expressed as the mean \pm standard deviation of three independent experiments. Control, untransfected SKOV3 cells; NC, negative control SKOV3 cells transfected with scramble miRNA; miR, microRNA; FITC, fluorescein isothiocyanate.

A
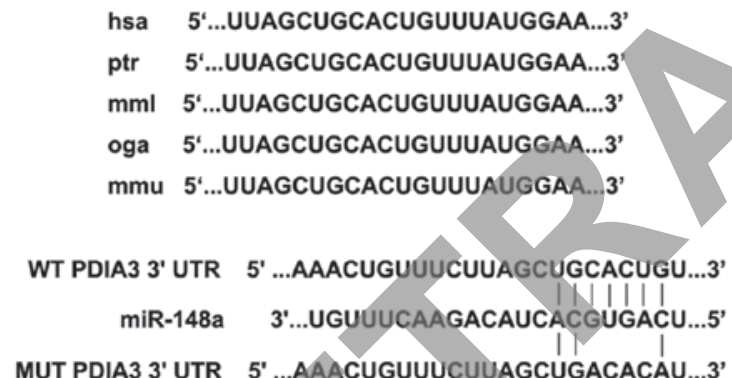

C

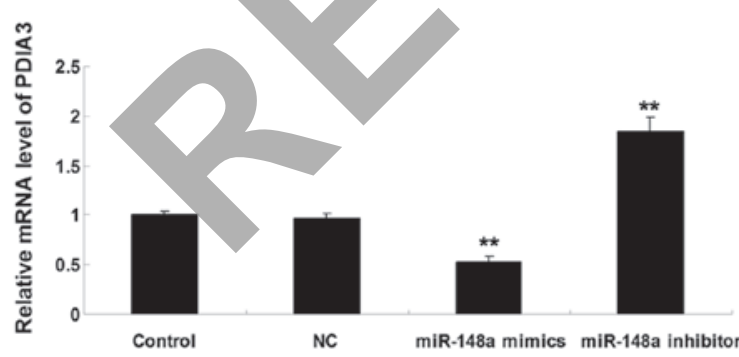

B

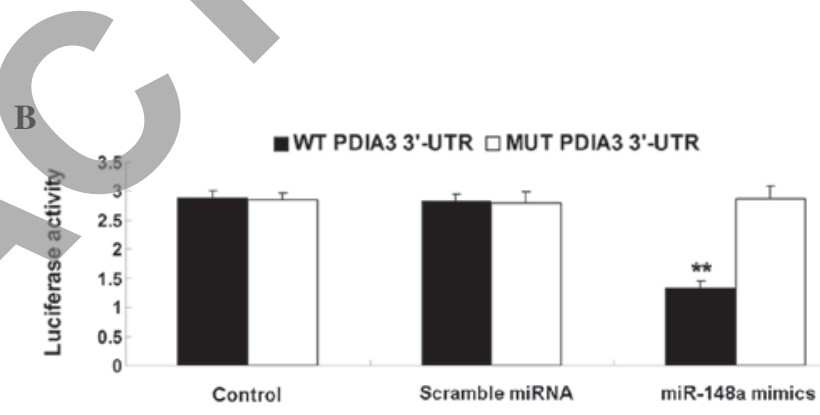

D

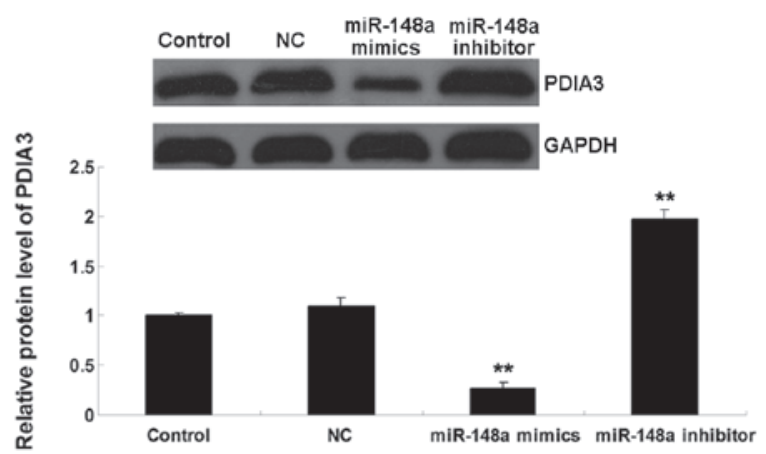

Figure 2. (A) As predicted by TargetScan, the 3'UTR of PDIA3 contained the evolutionarily conversed binding site of miR-148a. The WT and MUT PDIA3 3'-UTRa are shown. (B) A luciferase reporter assay demonstrated that the luciferase activity was significantly reduced in the SKOV3 human ovarian cancer cells co-transfected with the WT PDIA3 3'UTR and miR-148a mimic. However, the luciferase activity was unchanged in the SKOV3 cells co-transfected with the MUT PDIA3 3'UTR and miR-148a mimic ( ${ }^{* *} \mathrm{P}<0.0,1$ vs. control). (C) mRNA expression levels of PDIA3 were significantly reduced following overexpression of miR-148a, but were increased following inhibition of miR-148a in the SKOV3 cells ( ${ }^{* *} \mathrm{P}<0.01$, vs. control). (D) Protein expression levels of PDIA3 were significantly reduced following overexpression of miR-148a, but increased following inhibition of miR-148a in the SKOV3 cells ( ${ }^{* *} \mathrm{P}<0.01$, vs. control). The results are expressed as the mean \pm standard deviation of three independent experiments. Control, untransfected SKOV3 cells; NC, negative control SKOV3 cells transfected with scramble miRNA; miR, microRNA; UTR, untranslated region; PDIA3, protein disulfide isomerase family A, member 3.

respectively. RT-qPCR and western blotting were performed following transfection and demonstrated that the mRNA and protein expression levels of PDIA3 were significantly upregulated in the SKOV3 cells transfected with the pcDNA3.1-PDIA3 plasmid $(\mathrm{P}<0.01)$, however, the levels were reduced in the SKOV3 cells transfected with PDIA3 siRNA 
A

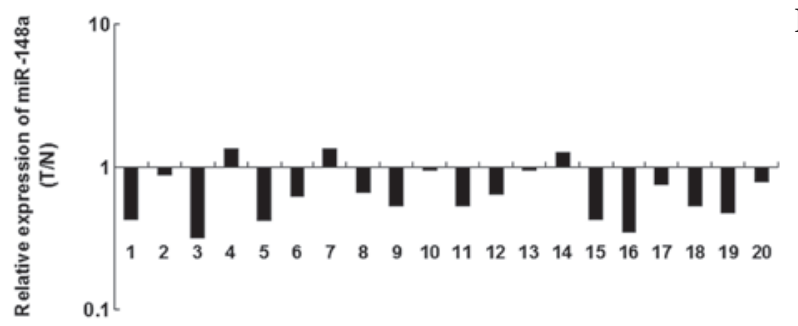

B

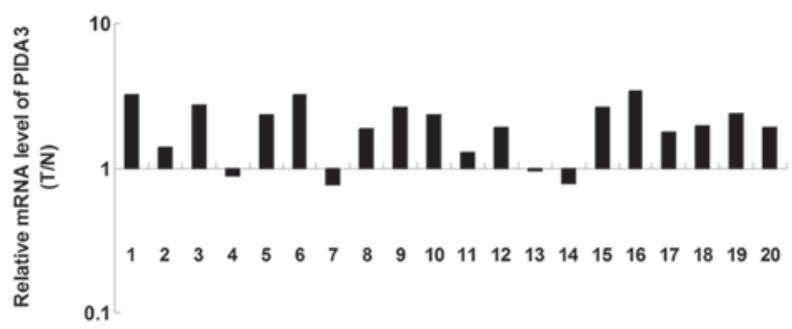

C

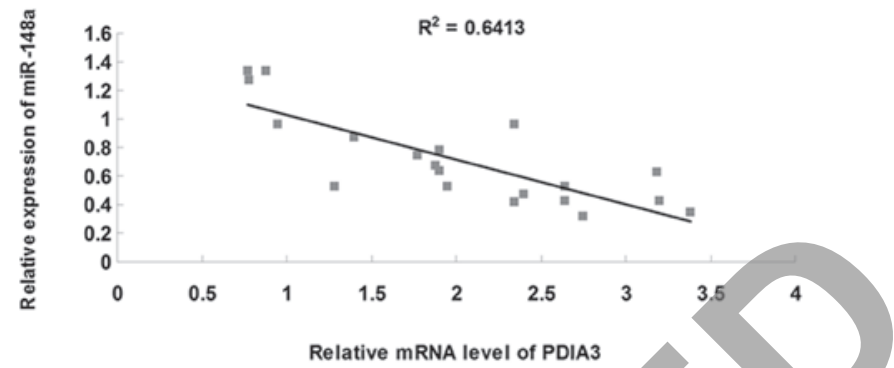

Figure 3. (A) RT-qPCR data demonstrated that miR-148a was significantly downregulated in ovarian cancer tissue (T), compared with matched normal adjacent tissue (N). (B) RT-PCR data demonstrated that the mRNA expression levels of PDIA3 were markedly increased in ovarian cancer tissue, compared with normal adjacent tissue. (C) A significant inverse correlation was detected between the expression levels of miR-148a and PDIA3 in the ovarian cancer tissues. The results are expressed as the mean \pm standard deviation of three independent experiments. miR, microRNA; RT-qPCR, reverse transcription-quantitative polymerase chain reaction; PDIA3 protein disulfide isomerase family A, member 3.

A

C

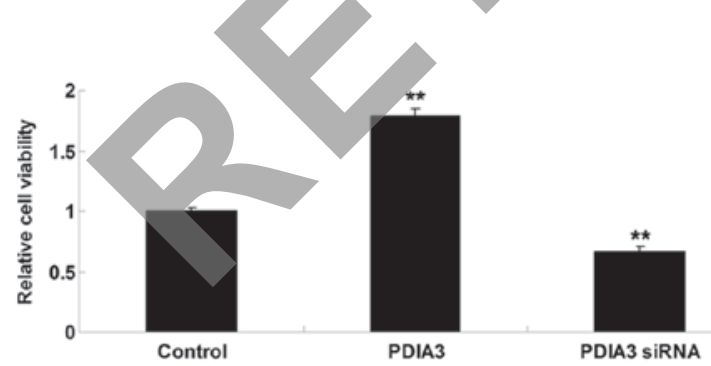

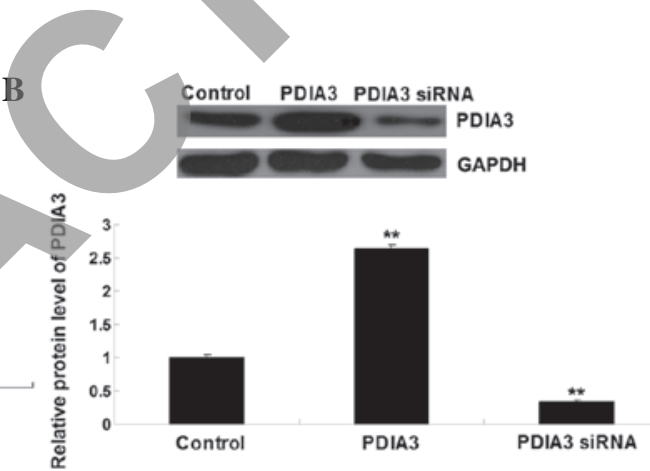

D
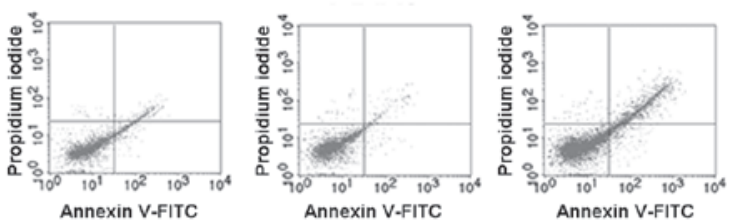

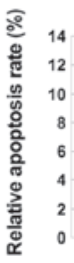
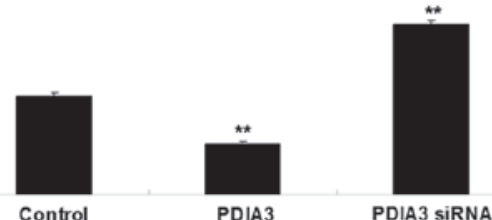

Figure 4. (A) mRNA expression levels of PDIA3 were significantly upregulated in SKOV3 human ovarian cancer cells transfected with pcDNA3.1-PDIA3, but reduced in SKOV3 cells transfected with PDIA3 siRNA ("P $<0.01$, vs. control). (B) Protein expression levels of PDIA3 were significantly upregulated in SKOV3 cells transfected with pcDNA3.1-PDIA3, but were reduced in SKOV3 cells transfected with PDIA3 siRNA ("P<0.01 vs. control). (C) MTT assay demonstrated that upregulation of PDIA3 promoted viability, whereas inhibition of PDIA3 inhibited viability of the SKOV3 cells, compared with the control cells ("*P<0.01, vs. control). (D) Overexpression of PDIA3 inhibited paclitaxel-induced cell apoptosis, whereas inhibition of PDIA3 promoted paclitaxel-induced cell apoptosis, compared with the control (" $\mathrm{P}<0.01$, vs. control). The results are expressed as the mean \pm standard deviation of three independent experiments. Control, untransfected SKOV3 cells; PDIA3, protein disulfide isomerase family A, member 3; siRNA, small interfering RNA; FITC, fluorescien isothiocyanate.

$(\mathrm{P}<0.01)$ (Figs. 4A and B). Subsequently, the cell viability of each group was determined using an MTT assay. Upregulation of PDIA3 promoted cell viability, whereas inhibition of PDIA3 inhibited the viability of the SKOV3 cells, compared with the untransfected control cells $(\mathrm{P}<0.01)$ (Fig. 4C). Furthermore, the SKOV3 cells were treated with paclitaxel, in order to 
induce cell apoptosis, and the percentage of apoptotic cells was detected $48 \mathrm{~h}$ after transfection. As shown in Fig. 4D, overexpression of PDIA3 inhibited paclitaxel-induced cell apoptosis $(\mathrm{P}<0.01)$, whereas inhibition of PDIA3 promoted paclitaxel-induced cell apoptosis, compared with the control group $(\mathrm{P}<0.01)$. These results indicated that PDIA3 inhibited the paclitaxel-induced apoptosis of ovarian cancer cells.

\section{Discussion}

It has been suggested that miR-148a is associated with human ovarian cancer (12). However, the detailed role of miR-148a in ovarian cancer, as well as the underlying mechanism, remain to be fully elucidated. The present study demonstrated that miR-148a inhibited the proliferation and promoted the paclitaxel-induced apoptosis of ovarian cancer cells. However, the effects of miR-148a on cancer cell proliferation remain controversial. A previous study demonstrated that miR-148a, as an androgen-responsive miR, promotes prostate cancer cell growth by repressing the expression of CAND1 (16). However, miR-148a has also been reported to have a suppressive role in the regulation of cancer cell proliferation by targeting different genes $(17,18)$. These findings suggest that the effects of miR-148a on cancer cell proliferation are tumor-specific. Furthermore, miR-148a has been demonstrated to attenuate the paclitaxel resistance of hormone-refractory, drug-resistant prostate cancer cells, through directly targeting MSK1 (19). However, whether other miR-148a targets are associated with paclitaxel resistance remains to be elucidated.

The present study aimed to investigate the potential target genes of miR-148a by performing bioinformatics prediction analysis, and identified PDIA3 as a target gene of miR-148a. Further investigation revealed that miR-148a was able to negatively regulate the mRNA and protein expression levels of PDIA3 by binding directly to the 3'-UTR of PDIA3 mRNA in SKOV3 ovarian cancer cells. PDIA3, also termed ER-60 or ERp57, is an endoplasmic reticulum protein that possesses protein disulfide isomerase activity and interacts with calreticulin and calnexin lectin chaperones to modulate the folding of newly synthesized glycoproteins (20). The role of PDIA3 in the development and progression of human cancer has been suggested previously. Downregulation of the expression of PDIA3 is associated with a poor prognosis in early-stage cervical cancer (21). In addition, PDIA3 was found to contribute to epidermal growth factor receptor signaling and internalization in breast cancer cells (22). A previous study demonstrated that PDIA3 is involved in paclitaxel resistance in ovarian cancer by interacting with class III $\beta$-tubulin (23). Accordingly, it is possible that miR-148a/PDIA3 is involved in the regulation of paclitaxel resistance in ovarian cancer.

The present study examined the expression levels of miR-148a and PDIA3 in ovarian cancer tissue samples. miR-148a was frequently downregulated in ovarian cancer tissue, whereas the expression levels of PDIA3 were increased in ovarian cancer tissues, compared with matched adjacent normal tissues. Furthermore, a significant inverse correlation was detected between the expression levels of miR-148a and PDIA3 in ovarian cancer tissues. These results indicated that downregulation of miR-148a contributes to the inhibition of PDIA3 in ovarian cancer.
The present study also demonstrated that knockdown of PDIA3 significantly inhibited proliferation and promoted paclitaxel-induced apoptosis of ovarian cancer cells, whereas overexpression of PDIA3 had the opposite effects. Therefore, PDIA3 was suggested as another key target of miR-148a that is closely associated with paclitaxel resistance in ovarian cancer cells. Lwin et al (24) observed that downregulation of PDIA3 by siRNA significantly inhibited cell proliferation by inducing $\mathrm{G}_{1} / \mathrm{S}$ arrest in breast cancer cells, whereas Cicchillitti et al (25) demonstrated that the interaction of nuclear PDIA3 with $\beta$-actin is associated with paclitaxel resistance in epithelial ovarian cancer cells, and that specific actin conformations modulate this complex (25). Based on the findings of these previous studies and of the present study, it was suggested that PDIA3 not only contributes to paclitaxel resistance, but also promotes ovarian cancer growth.

In conclusion, the present study demonstrated that miR-148a inhibited the expression of PDIA3 by targeting the 3'-UTR of PDIA3 mRNA. By mediating the expression of PDIA3, miR-148a inhibited the proliferation and promoted the paclitaxel-induced apoptosis of ovarian cancer cells. The results of the present study may provide a novel insight into the growth of ovarian cancer cells, and suggest that miR-148a may serve as a diagnostic and therapeutic marker in ovarian cancer.

\section{References}

1. Choi JH, Wong AS, Huang HF and Leung PC: Gonadotropins and ovarian cancer. Endocr Rev 28: 440-461, 2007.

2. Shylasree TS, Bryant A and Athavale R: Chemotherapy and/or radiotherapy in combination with surgery for ovarian carcinosarcoma. Cochrane Database Syst Rev 2: CD006246, 2013.

3. Llauradó M, Majem B, Altadill T, et al: MicroRNAs as prognostic markers in ovarian cancer. Mol Cell Endocrinol 390: 73-84, 2014.

4. Park SH, Song JY, Kim YK, et al: Fascin1 expression in high-grade serous ovarian carcinoma is a prognostic marker and knockdown of fascin 1 suppresses the proliferation of ovarian cancer cells. Int J Oncol 44: 637-646, 2014.

5. Ambros V: The functions of animal microRNAs. Nature 431: 350-355, 2004.

6. Bartel DP: MicroRNAs: Genomics, biogenesis, mechanism and function. Cell 116: 281-297, 2004.

7. Bienertova-Vasku J, Sana J and Slaby O: The role of microRNAs in mitochondria in cancer. Cancer Lett 336: 1-7, 2013.

8. Li J, Song Y, Wang Y, Luo J and Yu W: MicroRNA-148a suppresses epithelial-to-mesenchymal transition by targeting ROCK1 in non-small cell lung cancer cells. Mol Cell Biochem 380: 277-282, 2013.

9. Heo MJ, Kim YM, Koo JH, et al: microRNA-148a dysregulation discriminates poor prognosis of hepatocellular carcinoma in association with USP4 overexpression. Oncotarget 5: 2792-2806, 2014.

10. Zheng B, Liang L, Wang C, et al: MicroRNA-148a suppresses tumor cell invasion and metastasis by downregulating ROCK1 in gastric cancer. Clin Cancer Res 17: 7574-7583, 2011.

11. Zhang JP, Zeng C, Xu L, Gong J, Fang JH and Zhuang SM: MicroRNA-148a suppresses the epithelial-mesenchymal transition and metastasis of hepatoma cells by targeting Met/Snail signaling. Oncogene 33: 4069-4076, 2014.

12. Zhou X, Zhao F, Wang ZN, et al: Altered expression of miR-152 and miR-148a in ovarian cancer is related to cell proliferation. Oncol Rep 27: 447-454, 2012.

13. Hou F, Wang L, Wang H, et al: Elevated gene expression of S100A12 is correlated with the predominant clinical inflammatory factors in patients with bacterial pneumonia. Mol Med Rep 11: 4345-4352, 2015.

14. Chay D, Cho H, Lim BJ, et al: ER-60 (PDIA3) is highly expressed in a newly established serous ovarian cancer cell line, YDOV-139. Int J Oncol 37: 399-412, 2010.

15. Ménoret A, Drew DA, Miyamoto S, et al: Differential proteomics identifies PDIA3 as a novel chemoprevention target in human colon cancer cells. Mol Carcinog 53 (Suppl 1): E11-E22, 2014. 
16. Murata T, Takayama K, Katayama S, et al: $\operatorname{miR}-148$ a is an androgen-responsive microRNA that promotes LNCaP prostate cell growth by repressing its target CAND1 expression. Prostate Cancer Prostatic Dis 13: 356-361, 2010.

17. Xia J, Guo X, Yan J and Deng K: The role of miR-148a in gastric cancer. J Cancer Res Clin Oncol 140: 1451-1456, 2014.

18. Long XR, He Y, Huang C and Li J: MicroRNA-148a is silenced by hypermethylation and interacts with DNA methyltransferase 1 in hepatocellular carcinogenesis. Int J Oncol 44: 1915-1922, 2014.

19. Fujita Y, Kojima K, Ohhashi R, et al: MiR-148a attenuates paclitaxel resistance of hormone-refractory, drug-resistant prostate cancer PC 3 cells by regulating MSK1 expression. J Biol Chem 285: 19076-19084, 2010.

20. Turano C, Gaucci E, Grillo C and Chichiarelli S: ERp57/GRP58: A protein with multiple functions. Cell Mol Biol Lett 16: 539-563, 2011.

21. Chung H, Cho H, Perry C, et al: Downregulation of ERp57 expression is associated with poor prognosis in early-stage cervical cancer. Biomarkers 18: 573-579, 2013.
22. Gaucci E, Altieri F, Turano C and Chichiarelli S: The protein ERp57 contributes to EGF receptor signaling and internalization in MDA-MB-468 breast cancer cells. J Cell Biochem 114: 2461-2470, 2013.

23. Cicchillitti L, Di Michele M, Urbani A, et al: Comparative proteomic analysis of paclitaxel sensitive A2780 epithelial ovarian cancer cell line and its resistant counterpart A2780TC1 by 2D-DIGE: The role of ERp57. J Proteome Res 8: 1902-1912, 2009.

24. Lwin ZM, Yip GW, Chew FT and Bay BH: Downregulation of ER60 protease inhibits cellular proliferation by inducing $\mathrm{G}_{1} / \mathrm{S}$ arrest in breast cancer cells in vitro. Anat Rec (Hoboken) 295: 410-416, 2012.

25. Cicchillitti L, Della Corte A, Di Michele M, Donati MB, Rotilio D and Scambia G: Characterisation of a multimeric protein complex associated with ERp57 within the nucleus in paclitaxel-sensitive and -resistant epithelial ovarian cancer cells: The involvement of specific conformational states of beta-actin. Int J Oncol 37: 445-454, 2010. 